\title{
Imaging of Platelet-Derived Growth Factor Receptor $\beta$ Expression in Glioblastoma Xenografts Using Affibody Molecule 111In-DOTA-Z09591
}

\author{
Vladimir Tolmachev ${ }^{1}$, Zohreh Varasteh ${ }^{2}$, Hadis Honarvar ${ }^{1}$, Seyed Jalal Hosseinimehr ${ }^{1,3}$, Olof Eriksson ${ }^{2}$, Per Jonasson ${ }^{4}$, \\ Fredrik Y. Frejd ${ }^{1,4}$, Lars Abrahmsen ${ }^{5}$, and Anna Orlova ${ }^{2}$ \\ ${ }^{I}$ Division of Biomedical Radiation Sciences, Rudbeck Laboratory, Uppsala University, Uppsala, Sweden; ${ }^{2}$ Preclinical PET Platform, \\ Department of Medicinal Chemistry, Uppsala University, Uppsala, Sweden; ${ }^{3}$ Department of Radiopharmacy, Faculty of Pharmacy, \\ Mazandaran University of Medical Sciences, Sari, Iran; ${ }^{4}$ Affibody AB, Stockholm, Sweden; and ${ }^{5}$ Algeta ASA, Oslo, Norway
}

\begin{abstract}
The overexpression and excessive signaling of platelet-derived growth factor receptor $\beta$ (PDGFR $\beta$ ) has been detected in cancers, atherosclerosis, and a variety of fibrotic diseases. Radionuclide in vivo visualization of PDGFR $\beta$ expression might help to select PDGFR $\beta$ targeting treatment for these diseases. The goal of this study was to evaluate the feasibility of in vivo radionuclide imaging of PDGFR $\beta$ expression using an Affibody molecule, a small nonimmunoglobulin affinity protein. Methods: The PDGFR $\beta$-binding Z09591 Affibody molecule was site-specifically conjugated with a maleimido derivative of DOTA and labeled with ${ }^{111} \mathrm{In}$. Targeting of the PDGFR $\beta$-expressing U-87 MG glioblastoma cell line using ${ }^{111}$ In-DOTA-Z09591 was evaluated in vitro and in vivo. Results: DOTA-Z09591 was stably labeled with ${ }^{111} \mathrm{In}$ with preserved specific binding to PDGFR $\beta$-expressing cells in vitro. The dissociation constant for ${ }^{111}$ In-DOTA-Z09591 binding to U-87 MG cells was determined to be $92 \pm 10 \mathrm{pM}$. In mice bearing U-87 MG xenografts, the tumor uptake of ${ }^{111}$ In-DOTA-Z09591 was $7.2 \pm 2.4$ percentage injected dose per gram and the tumor-to-blood ratio was $28 \pm 14$ at $2 \mathrm{~h}$ after injection. In vivo receptor saturation experiments demonstrated that targeting of U-87 MG xenografts in mice was PDGFR $\beta$-specific. U-87 MG xenografts were clearly visualized using small-animal SPECT/CT at $3 \mathrm{~h}$ after injection. Conclusion: This study demonstrates the feasibility of in vivo visualization of PDGFR $\beta$ expressing xenografts using an Affibody molecule. Further development of radiolabeled Affibody molecules might provide a useful clinical imaging tool for PDGFR $\beta$ expression during various pathologic conditions.

Key Words: Affibody molecule; ${ }^{111} \mathrm{In}$; molecular imaging; PDGFR $\beta$; U-87 MG xenograft
\end{abstract}

J Nucl Med 2014; 55:294-300

DOI: 10.2967/jnumed.113.121814

$\mathbf{T}$ he platelet-derived growth factor (PDGF) signaling axis includes 5 ligands composed of disulfide-bonded dimers of A, B, C, and $\mathrm{D}$ polypeptide chains as well as an $\mathrm{AB}$ heterodimer and 2 plateletderived growth factor receptors, PDGFR $\alpha$ and PDGFR $\beta$, which are receptor tyrosine kinases (1). Activation of PDGF receptors by ligand

Received Feb. 19, 2013; revision accepted Oct. 21, 2013.

For correspondence or reprints contact: Vladimir Tolmachev, Biomedical Radiation Sciences, Uppsala University, SE-75185, Uppsala, Sweden.

E-mail: Vladimir.Tolmachev@bms.uu.se

Published online Jan. 9, 2014.

COPYRIGHT (C) 2014 by the Society of Nuclear Medicine and Molecular Imaging, Inc. binding triggers intracellular signaling cascade, resulting in cell migration, survival, and proliferation (2). PDGF-A and PDGF-B, and their receptors, PDGFR $\alpha$ and PDGFR $\beta$, are expressed in a largely nonoverlapping pattern, suggesting a paracrine mode of action. PDGF-B and PDGFR $\beta$ are mainly expressed in developing vasculature, where PDGF-B is produced by endothelial cells and PDGFR $\beta$ is expressed by mural cells (i.e., pericytes and vascular smooth muscle cells) (3). In the adult, PDGF signaling stimulates wound healing and regulates the interstitial fluid pressure of tissues (4).

Excessive signaling of PDGF has been found in diseases involving excessive cell proliferation, such as cancer, atherosclerosis, and various fibrotic conditions (4). PDGFs and their receptors play an important role in many cancers including lung cancer, prostate cancer, renal cell carcinoma, chronic monomyelocytic leukemia, and glioblastoma (5). PDGFR activation can stimulate tumor growth directly by autocrine stimulation. Paracrine stimulation of PDGFR $\beta$ on tumor blood vessels pericytes and stromal fibroblasts contributes to tumor growth and homeostasis (4). Not surprisingly, expression of PDGFR $\beta$ is a prognostic biomarker for more aggressive disease. In breast and prostate cancer, stromal PDGFR $\beta$ expression correlates with shorter survival $(6,7)$. Increased expression of PDGFR $\beta$ is also associated with poor overall survival in angiosarcoma (8), hepatocellular carcinoma (9), and a subset of non-small cell lung cancers $(10)$.

Blocking PDGFR $\beta$ signaling reduces interstitial fluid pressure in tumors and improves uptake of antitumor drugs (4). PDGFR $\beta$ is one of the targets for tyrosine kinase inhibitors (TKI), such as imatinib, sunitinib, and sorafenib. The use of these TKIs improves survival of patients with hepatocellular carcinoma, gastrointestinal stromal tumors, and renal cell carcinoma. Although TKIs generally target several tyrosine kinases, research suggests that PFDGR $\beta$ is a key target for imatinib in several malignancies (11-13). There is a well-recognized unmet clinical need for biomarkers for selection of patients who might benefit from such therapy (14). Furthermore, there is a need for biomarkers for the detection of a drug effect on the target (i.e., pharmacodynamic biomarkers) and for the detection of onset of resistance in initial responders.

The histopathologic evaluation of biopsy samples is considered as a gold standard in the determination of relevant molecular targets and in monitoring of response to therapy. However, morbidity associated with invasive biopsies limits their use. In addition, spatial and temporal heterogeneity in the expression of molecular targets reduces the diagnostic value of biopsies. Radionuclide molecular 
imaging is an alternative to biopsies, permitting the visualization of molecular events by a noninvasive procedure.

Currently, the response to many TKIs is monitored indirectly, for example, by measuring changes in ${ }^{18} \mathrm{~F}$-FDG uptake in gastrointestinal stromal tumors treated by imatinib (15). This method provides information mainly about metabolism in the lesion and its changes in response to therapy. Imaging of receptor tyrosine kinase expression might provide important additional diagnostic information that cannot be obtained from ${ }^{18} \mathrm{~F}-\mathrm{FDG}$ imaging. The development of radionuclide-labeled tracers for the visualization of receptor tyrosine kinases is therefore one focus in radiopharmaceutical research (16-19). Radiolabeled TKIs have the potential advantage of enabling direct imaging of the therapeutic target. The synthesis of the ${ }^{11} \mathrm{C}$-labeled PDGFR $\beta$-targeting TKIs sorafenib (20), sunitinib (21), and imatinib (22) has been reported. However, experiments in baboons demonstrated that ${ }^{11} \mathrm{C}$-imatinib yields high accumulation of radioactivity in liver and gallbladder (22), reflecting a general problem of radiolabeled TKI. Imaging of intracellular targets requires that a tracer can not only permeate cellular membranes before binding to its target and but also is efficiently washed away from nonspecific compartments lacking the target. The penetration of cellular membranes requires elevated hydrophobicity. However, hydrophobicity is usually associated with an elevated level of hepatic uptake and hepatobiliary excretion (23). Indeed, a high hepatic uptake (exceeding tumor uptake) has been found in all in vivo studies with radiolabeled TKIs. Thus, the use of radiolabeled TKIs might be restricted to imaging of extrahepatic metastases. This is a serious drawback because the liver is a major metastatic site for many cancers.

Imaging of the extracellular domain of PDGFR $\beta$ might be a superior alternative to radiolabeled TKIs. A new class of nonimmunoglobulin-based targeting proteins, Affibody molecules (Affibody AB, Sweden), have been used for the visualization of several extracellular molecular targets (24). For example, radiolabeled anti-human epidermal growth factor receptor (HER2) Affibody molecules have provided high-contrast imaging of HER2-expressing tumors a few hours after injection both in preclinical (25) and clinical studies $(26,27)$. Recently, we have developed the Affibody molecule Z02465, showing high-affinity binding to both human and murine PDGFR $\beta\left(\mathrm{K}_{\mathrm{D}}=0.5\right.$ and $7.1 \mathrm{nM}$, respectively) (28). This Affibody molecule competes with the natural ligand, PDGF-BB, for binding to PDGFR $\beta$ and inhibits ligand-induced receptor phosphorylation (28).

We hypothesized that radiolabeled derivatives of the Affibody molecule Z02465 would be suitable for radionuclide imaging of PDGFR $\beta$ expression in vivo. To test this hypothesis, the Z02465 was redesigned. A unique thiol group was created by introducing a cysteine at the $\mathrm{C}$ terminus, enabling site-specific labeling using thiol-directed chemistry. In addition, the first 2 amino acids at the $\mathrm{N}$ terminus were changed from VD- to AE-, because such substitution improves biodistribution of Affibody molecules (29). The resulting variant of denoted Z09591 was labeled with ${ }^{111}$ In and evaluated for targeting of PDGFR $\beta$-expressing U-87 MG cells in vitro and in vivo.

\section{MATERIALS AND METHODS}

\section{Statistics}

Data on cellular uptake and biodistribution were analyzed by unpaired, 2-tailed $t$ test using GraphPad Prism (version 4.00 for Windows; GraphPad Software) to determine significant differences $(P<$ $0.05)$.

\section{Affibody Molecule}

The Affibody molecule Z09591-Cys was expressed in Escherichia coli and purified essentially as described earlier (30). The maleimido derivative of the DOTA chelator (Macrocyclics) was conjugated to the cysteine thiol of Z09591 as described earlier (30). The identity of the conjugate (designated as DOTA-Z09591) was confirmed and the purity evaluated by high-performance liquid chromatography and in-line mass spectrometry (HPLC-MS) using an Agilent 1100 HPLC/MSD equipped with electrospray ionization and a single quadrupole as described earlier (30). A stock solution of DOTA-Z09591 $(1.32 \mathrm{mg} / \mathrm{mL}$ in $0.2 \mathrm{M}$ sodium acetate, $\mathrm{pH}$ 5.4) was stored frozen.

\section{Labeling and Stability Assessment}

For labeling of DOTA-Z09591, $40 \mu \mathrm{L}$ of stock solution $(52.8 \mu \mathrm{g}$ of protein) were mixed with $40 \mu \mathrm{L}$ of $0.2 \mathrm{M}$ ammonium acetate, $\mathrm{pH} 5.5$, and $80 \mu \mathrm{L}$ of ${ }^{111}$ In stock solution (30-60 MBq). The reaction mixtures were incubated at $80^{\circ} \mathrm{C}$ for $30 \mathrm{~min}$ and thereafter analyzed by radio-instant thin-layer chromatography (ITLC). To cross-validate the ITLC results, a sodium dodecyl sulfate polyacrylamide gel electrophoresis (SDS-PAGE) analysis and size-exclusion chromatography were performed (supplemental data; supplemental materials are available at http://jnm.snmjournals. org). To evaluate the stability of labeling, the ${ }^{111}$ In-labeled conjugates were incubated with a 500-fold excess of ethylenediaminetetraacetic acid (EDTA) for $4 \mathrm{~h}$ at room temperature and then analyzed using ITLC. Stability in human blood plasma was analyzed using size-exclusion chromatography, as described in the supplemental data.

\section{In Vitro Cell Binding and Processing of ${ }^{111}$ In-Labeled Affibody Molecules}

The PDGFR $\beta$-expressing U-87 MG glioma cell line (American Type Culture Collection) was used for binding specificity and cellular processing studies. Details concerning culturing and seeding are provided in the supplemental data.

An in vitro specificity test was performed, and the rate of internalization of ${ }^{111}$ In-DOTA-Z09591 by U-87 MG cells was evaluated according to the method described earlier (31). Experimental details are provided in the supplemental data.

The affinity of ${ }^{111}$ In-DOTA-Z09591 to U-87 MG cells and the number of binding sites were determined using LigandTracer (Ridgeview Instruments AB, Sweden) (32). Experimental details are provided in the supplemental data. The measurements were repeated 6 times at room temperature. Two additional measurements were performed at $4^{\circ} \mathrm{C}$.

\section{In Vivo Evaluation of ${ }^{111}$ In-DOTA-Z09591}

All animal experiments were planned and performed in accordance with national legislation on laboratory animals' protection and were approved by the Ethics Committee for Animal Research in Uppsala. Female BALB/C nu/nu mice were from Taconic M\&B, 6-8 wk old at arrival. For xenografting, $2 \times 10^{6} \mathrm{U}-87 \mathrm{MG}$ cells in phosphate-buffered saline were subcutaneously implanted in the right hind leg. At the time of biodistribution experiments ( $3 \mathrm{wk}$ after tumor implantation), the average mouse weight was $19.9 \pm 0.9 \mathrm{~g}$.

To study the influence of injected DOTA-Z09591 protein dose on targeting, the mice were divided into 4 groups, 4 animals each, and injected with $30 \mathrm{kBq}$ of ${ }^{111}$ In-DOTA-Z09591 in $100 \mu \mathrm{L}$ of phosphatebuffered saline. The average tumor weight was $0.34 \pm 0.1 \mathrm{~g}$. The injected protein dose was adjusted to $0.1,0.5,1$, or $70 \mu \mathrm{g}$ by the addition of nonlabeled DOTA-Z09591. At $2 \mathrm{~h}$ after injection, mice were sacrificed by exsanguination under anesthesia. Tumors as well as samples of tissues were collected and weighed, and their radioactivity was measured. The whole gastrointestinal tract (GI tract), with the exception of a small segment of colon, was taken together with its content to assess hepatobiliary excretion. Tissue uptake was calculated as percentage injected dose per gram $(\% \mathrm{ID} / \mathrm{g})$. Radioactivity in the 
gastrointestinal tract was calculated as percentage injected dose (\%ID) per whole sample.

An additional experiment was performed to assess the potential effects of high doses of the PDGFR $\beta$-specific DOTA-Z09591 on the biodistribution of any type of Affibody molecules. The effect of coinjection of $70 \mu \mathrm{g}$ of DOTA-Z09591 on the biodistribution of nonPDGFR $\beta$-specific (HER2-binding) ${ }^{111}$ In-DOTA-Z02395 Affibody molecule was studied in female non-tumor-bearing BALB/C nu/nu mice. In the test group, ${ }^{111}$ In-DOTA-Z02395 $(30 \mathrm{kBq}, 1 \mu \mathrm{g})$ was coinjected with $70 \mu \mathrm{g}$ of DOTA-Z09591. In the control groups, only the ${ }^{111}$ In-DOTA-Z02395 Affibody molecule $(30 \mathrm{kBq})$ was injected at protein doses of 1 and 70 $\mu \mathrm{g}$ per mouse. The biodistribution was measured at $2 \mathrm{~h}$ after injection as described above.

The biodistribution of ${ }^{111} \mathrm{In}$-DOTA-Z09591 at 1,2 , and $4 \mathrm{~h}$ in mice bearing U-87 MG xenografts was investigated at an injected dose of $1 \mu \mathrm{g}$. The mice were randomized into 3 groups, 4 animals each, and injected with $30 \mathrm{kBq}$ of ${ }^{111}$ In-DOTA-Z09591 in $100 \mu \mathrm{L}$ of phosphatebuffered saline and sacrificed at predetermined time points. The biodistribution was measured at predetermined time points as described above.

In the imaging experiment, a U-87 MG xenograft-bearing mouse was injected with $1 \mathrm{MBq}$ (amount of peptide, $1 \mu \mathrm{g}$ ) of ${ }^{111} \mathrm{In}$-DOTAZ09591. Immediately before imaging, $3 \mathrm{~h}$ after injection, the mouse was sacrificed. The SPECT/CT imaging experiment was performed using the Triumph Trimodality system (Gamma Medica), an integrated smallanimal SPECT/PET/CT platform. A CT image (field of view, $53.82 \mathrm{~mm}$, 512 projections; scanning time, $2.13 \mathrm{~min}$ ) was acquired to position the body of the animal in the scanner and for assisting in image analysis. SPECT acquisition was performed using 2 solid-state cadmium zinc telluride camera heads equipped with 5-pinhole 75A10 collimators. The following acquisition parameters were used for SPECT: nongated; field of view, $53.82 \mathrm{~mm}$; tomographic; 32 projections; $1,000 \mathrm{~s}$. SPECT raw data were reconstructed with FLEX SPECT software (Gamma Medica), which uses an ordered-subset expectation maximization iterative reconstruction algorithm. CT raw files were reconstructed by filtered backprojection. SPECT and CT data were fused and analyzed in PMOD (PMOD Technologies).

\section{RESULTS}

\section{Labeling and Stability Assessment}

According to mass-spectrometry analysis, $95 \%$ of the Affibody molecules were conjugated with a single DOTA chelator. Five percent remained unconjugated and exist in dimeric form. The radiochemical yield of ${ }^{111} \mathrm{In}$-DOTA-Z09591 was $99.3 \% \pm 0.6 \%$ (specific radioactivity $\leq 1.1 \mathrm{MBq} / \mu \mathrm{g}$ ) after labeling at $80^{\circ} \mathrm{C}$ for $30 \mathrm{~min}$. Both radio-SDS-PAGE and size-exclusion chromatography analysis showed a radioactivity peak corresponding to Affibody molecules (Supplemental Figs. 1 and 2). A challenge with a 500-fold molar excess of EDTA during $4 \mathrm{~h}$ demonstrated high stability of the label. The radiochemical purity of the EDTA-treated samples was $96.6 \% \pm 0.4 \%$ whereas the purity of the untreated control was $97.8 \% \pm 0.4 \%$ - that is, the difference was within the accuracy of the analytic method. After 1- and 4-h incubation in human blood plasma, the Affibody-associated fraction of radioactivity was $99.2 \% \pm 0.6 \%$ and $97.3 \% \pm 1.1 \%$, respectively (Supplemental Fig. 2).

Because of the high purity, ${ }^{111}$ In-DOTA-Z09591 was used in further experiments without purification after labeling.

\section{In Vitro Cell Binding and Processing of ${ }^{111}$ In-Labeled Affibody Molecules}

The preincubation of PDGFR $\beta$-expressing U-87 MG glioma cells with nonlabeled conjugates significantly $(P<0.05)$ decreased the binding of the radiolabeled Affibody molecules, suggesting that the binding of ${ }^{111}$ In-DOTA-Z09591 to the receptor was saturable (Fig. 1A).

Data concerning the binding and internalization of ${ }^{111}$ In-DOTAZ09591 by PDGFR $\beta$-expressing U-87 MG glioma cell lines are presented in Figures $1 \mathrm{~B}$ and $1 \mathrm{C}$. The radioactivity in the alkaline fraction was $0.8 \% \pm 0.4 \%$ of the cell-associated radioactivity after incubation at $4^{\circ} \mathrm{C}$ (Fig. 1B), reflecting the accuracy of the method. The radioactivity in the alkaline fraction after incubation at $37^{\circ} \mathrm{C}$ was significantly higher, $7.6 \% \pm 0.4 \%$ of the cell-associated radioactivity. The kinetics of binding was rapid up to around $1 \mathrm{~h}$, then slower. The internalization of ${ }^{111}$ In-DOTA-Z09591 was relatively inefficient, with a slow increase of the internalized fraction to around $10 \%$ after $24 \mathrm{~h}$.

According the LigandTracer measurements, the association rate was $(2.0 \pm 0.3) \times 10^{5} \mathrm{M}^{-1} \mathrm{~s}^{-1}$ and dissociation rate $(1.8 \pm 0.4) \times$ $10^{-5} \mathrm{~s}^{-1}$. The dissociation constant for ${ }^{111}$ In-DOTA-Z09591 binding to living U-87 MG cells was $92 \pm 10 \mathrm{pM}$ at ambient temperature $\left(22^{\circ} \mathrm{C}\right)$ and $94 \pm 17 \mathrm{pM}$ at $4^{\circ} \mathrm{C}$. The cell line displayed $36,000 \pm 4,000$ binding sites per cell.

\section{In Vivo Evaluation of ${ }^{111}$ In-DOTA-Z09591}

Data concerning the biodistribution of ${ }^{111}$ In-DOTA-Z09591 in BALB/C $n u / n u$ mice bearing U-87 MG xenografts at $2 \mathrm{~h}$ after injection of $0.1,0.5,1$, or $70 \mu \mathrm{g}$ are presented in Table 1 . The biodistribution of ${ }^{111} \mathrm{In}-\mathrm{DOTA}-\mathrm{Z} 09591$ was characterized by a rapid disappearance from blood and normal tissues and build-up in the kidney, probably resulting from reabsorption in proximal tubuli after glomerular filtration. The accumulation of radioactivity in the gastrointestinal tract with its content (a measure for hepatobiliary excretion) was low $(<3 \%$ of injected radioactivity). At all protein doses, the uptake in tumor was appreciably higher than uptake in any other organ or tissue except for the kidney. Hepatic uptake was significantly higher after injection of $0.1 \mu \mathrm{g}$ than other doses. There were tendencies to decreasing uptake in the lung, stomach, and muscle with the increase injected protein dose from 0.1 to $1 \mu \mathrm{g}$ per mouse, but the difference was not significant. Similarly, a tendency of increased tumor uptake with increased dose in this range did not 
TABLE 1

Biodistribution of ${ }^{111}$ In-DOTA-Z09591 at 2 Hours After Injection in BALB/C nu/nu Mice Bearing U-87 MG Xenografts

\begin{tabular}{lcccc}
\hline & \multicolumn{4}{c}{ Total injected dose $(\mu \mathrm{g})$} \\
\cline { 2 - 5 } Organ & 0.1 & 0.5 & 1 & 70 \\
\hline Blood & $0.34 \pm 0.05$ & $0.35 \pm 0.07$ & $0.26 \pm 0.04$ & $0.14 \pm 0.02$ \\
\hline Lung & $0.8 \pm 0.2$ & $0.72 \pm 0.08$ & $0.7 \pm 0.2$ & $0.21 \pm 0.02$ \\
Liver & $0.99 \pm 0.07^{*, \dagger}$ & $0.84 \pm 0.07$ & $0.8 \pm 0.1$ & $0.39 \pm 0.05$ \\
Spleen & $1.8 \pm 0.4$ & $2.0 \pm 0.3$ & $1.6 \pm 0.2$ & $0.25 \pm 0.06$ \\
Stomach & $1.1 \pm 0.3$ & $1.0 \pm 0.2$ & $0.8 \pm 0.2$ & $0.19 \pm 0.02$ \\
Colon & $1.3 \pm 0.3$ & $1.5 \pm 0.2$ & $1.1 \pm 0.4$ & $0.23 \pm 0.05$ \\
Kidney & $288 \pm 15$ & $267 \pm 40$ & $256 \pm 19$ & $310 \pm 32$ \\
\hline Tumor & $5.6 \pm 1.1$ & $6.5 \pm 0.9$ & $7.2 \pm 2.4$ & $0.89 \pm 0.04$ \\
Muscle & $0.4 \pm 0.1$ & $0.4 \pm 0.1$ & $0.3 \pm 0.1$ & $0.07 \pm 0.02$ \\
Bone & $0.6 \pm 0.1$ & $0.8 \pm 0.2$ & $0.6 \pm 0.1$ & $0.14 \pm 0.05$ \\
Gl tract ${ }^{*}$ & $1.2 \pm 0.2$ & $1.7 \pm 0.7$ & $1.2 \pm 0.2$ & $0.50 \pm 0.13$ \\
\hline
\end{tabular}

*Significant difference $(P<0.05)$ between doses 0.1 and $0.5 \mu \mathrm{g}$.

${ }^{\dagger}$ Significant difference $(P<0.05)$ between doses 0.1 and $1 \mu \mathrm{g}$.

${ }^{\ddagger}$ Data for GI tract are presented as \%ID per whole sample.

Uptake in all organs and tissues, except kidneys, after injection of $70 \mu \mathrm{g}$ of ${ }^{111} \mathrm{In}$-DOTA-Z09591 was significantly lower than after injection of any other dose. Data are presented as average \%ID/g and SD for 4 mice.

reach significance. The tumor uptake $(5.6 \pm 1.1$ to $7.2 \pm 2.4$ $\% \mathrm{ID} / \mathrm{g}$ ) was in the expected range for Affibody molecules binding to a molecular target presented at this level. Increasing the injected protein dose to $70 \mu \mathrm{g}$ per mouse had a strong effect on the biodistribution (Table 1; Supplemental Fig. 4). Tumor uptake was reduced to $0.89 \pm 0.04 \% \mathrm{ID} / \mathrm{g}$, showing that uptake of ${ }^{111} \mathrm{In}-$ DOTA-Z09591 in tumor xenografts is saturable. There was also a significant decrease of uptake of other organs and tissues, except for the kidneys.

The coinjection of HER2-targeting ${ }^{111}$ In-DOTA-Z02395 Affibody molecule with $70 \mu \mathrm{g}$ of DOTA-Z09591 was performed to further elucidate the nature of this phenomenon, for example, potential effects on vascular permeability or glomerular filtration rate. The results are presented in Table 2. The coinjection of DOTA-Z09591 did not cause significant difference in uptake of the HER2-binding ${ }^{111}$ In-DOTA-Z02395 in any normal organ or tissue, except for the stomach and colon, in which uptake was increased. The coinjection of the same amount of cold DOTAZ02395 did not have any effect on ${ }^{111}$ In-DOTA-Z02395 uptake, in agreement with earlier data (27), suggesting that injection of $70 \mu \mathrm{g}$ of DOTA-Z09591 does not influence vascular permeability or excretion rate of Affibody molecules.

The data concerning tumor-to-organ ratios at $2 \mathrm{~h}$ after injection of $0.1,0.5$, and $1 \mu \mathrm{g}$ of ${ }^{111} \mathrm{In}$-MMA-DOTA-Z9591 in BALB/C $n u / n u$ mice bearing U-87 MG xenografts are presented in Table 3. There is a tendency of increased ratios with increased dose in this range. However, a significant difference was observed only for tumor-tostomach, tumor-to-colon, and tumor-to-muscle ratios between 0.1 and $1 \mu \mathrm{g}$ of injected protein. Because of a more favorable biodistribution profile, an injected dose of $1 \mu \mathrm{g}$ was selected for further studies.

The data concerning the biodistribution of ${ }^{111}$ In-DOTA-Z09591 in BALB/C $n u / n u$ mice bearing U-87 MG xenografts at 1,2 , and $4 \mathrm{~h}$ after injection of $1 \mu \mathrm{g}$ are presented in Table 4 . The data dem-

TABLE 2

Influence of Coinjection of DOTA-Z09591 on Biodistribution of Anti-HER2 ${ }^{111}$ In-DOTA-Z02395 at 2 Hours After Injection in BALB/C nu/nu Mice

\begin{tabular}{lccc}
\hline Organ & $\begin{array}{c}111 \mathrm{In}-\mathrm{DOTA}-Z 02395 \\
(1 \mu \mathrm{g})\end{array}$ & $\begin{array}{c}111 \mathrm{In}-\mathrm{DOTA}-Z 02395(1 \mu \mathrm{g})+70 \mu \mathrm{g} \\
\text { of DOTA-Z09591 }\end{array}$ & $\begin{array}{c}111 \mathrm{In}-\mathrm{DOTA}-Z 02395 \\
(70 \mathrm{~g})\end{array}$ \\
\hline Blood & $1.5 \pm 0.4$ & $1.2 \pm 0.1$ & $1.0 \pm 0.2$ \\
Lung & $1.8 \pm 0.3$ & $2.1 \pm 0.5$ & $1.8 \pm 0.2$ \\
Liver & $1.5 \pm 0.3$ & $1.7 \pm 0.1$ & $1.2 \pm 0.1$ \\
Spleen & $0.7 \pm 0.1$ & $0.8 \pm 0.2$ & $0.7 \pm 0.1$ \\
Stomach & $0.9 \pm 0.1$ & $1.3 \pm 0.2^{*}$ & $0.9 \pm 0.2$ \\
Colon & $0.9 \pm 0.1$ & $1.4 \pm 0.3^{*}$ & $0.83 \pm 0.06$ \\
Kidney & $238 \pm 32$ & $271 \pm 21$ & $215 \pm 35$ \\
Muscle & $0.47 \pm 0.08$ & $0.49 \pm 0.08$ & $0.43 \pm 0.06$ \\
Bone & $0.65 \pm 0.08$ & $0.77 \pm 0.09$ & $0.6 \pm 0.1$
\end{tabular}

*Significant difference $(P<0.05)$ between control and coinjection groups.

Data are presented as average \%ID/g and SD for 4 mice. 
TABLE 3

Tumor-to-Organ Ratios of ${ }^{111}$ In-DOTA-Z09591 Affibody Molecule (2 Hours After Injection) in BALB/C nu/nu Mice Bearing U-87 MG Xenografts

\begin{tabular}{lccc}
\hline & \multicolumn{3}{c}{ Dose $(\mu \mathrm{g})$} \\
\cline { 2 - 4 } \multicolumn{1}{c}{ Organ } & 0.1 & 0.5 & 1 \\
\hline Blood & $17 \pm 3$ & $19 \pm 5$ & $28 \pm 14$ \\
Lung & $7 \pm 2$ & $9 \pm 2$ & $12 \pm 4$ \\
Liver & $5.6 \pm 0.7$ & $8 \pm 2$ & $9 \pm 4$ \\
Spleen & $3.2 \pm 0.7$ & $3.3 \pm 0.5$ & $5 \pm 2$ \\
Stomach & $5 \pm 1^{*}$ & $7 \pm 2$ & $10 \pm 3$ \\
Colon & $4.2 \pm 0.7^{*}$ & $4 \pm 1$ & $6 \pm 1$ \\
Muscle & $13 \pm 3^{*}$ & $17 \pm 7$ & $28 \pm 7$ \\
Bone & $8.8 \pm 0.8$ & $9 \pm 3$ & $13 \pm 4$
\end{tabular}

*Significant difference $(P<0.05)$ between doses 0.1 and $1 \mu \mathrm{g}$. Data are presented as average of 4 animals and SD.

onstrate a rapid clearance from all organs and tissues except from the kidney. The radioactivity uptake in tumor was equal at 1 and $2 \mathrm{~h}$ after injection but decreased significantly at $4 \mathrm{~h}$ in comparison with $1 \mathrm{~h}$ after injection. Tumor-to-organ ratios (Table 5) appear to increase over time, although the only significant increase between 2 and $4 \mathrm{~h}$ was for the tumor-to-colon ratio. On the basis of these data, an optimal imaging time was determined to be between 2 and 4 after injection.

Small-animal SPECT/CT imaging, performed $3 \mathrm{~h}$ after injection (Fig. 2), confirmed the results of the biodistribution experiments. A substantial renal retention of radioactivity was visualized. Other organs and tissues were not seen. ${ }^{111}$ In-DOTA-Z09591 enabled imaging of the PDFGR $\beta$-expressing U-87 MG xenograft. Rapid clearance of the conjugates provided low background radioactivity and ensured a high imaging contrast.

\section{DISCUSSION}

A first imaging agent for noninvasive visualization of PDGFR $\beta$ expression is presented in this report. To our knowledge, no adequate imaging agent for PDGFR $\beta$ has previously been presented. PDGFR $\beta$ inhibition by imatinib has been assessed indirectly, by monitoring the tumor uptake of an anti-carcinoembryonic antigen antibody (33) or by studying the decrease of ${ }^{18} \mathrm{~F}$-FDG uptake (15). Askoxylakis et al. (34) have reported the selection and evaluation of unrestricted dodecapeptide-targeting PDGFR $\beta$. The reported affinity of this peptide is low, showing an $\mathrm{IC}_{50}$ (half maximal inhibitory concentration) of $1.4 \mu \mathrm{M}$. In vivo, the accumulation of radioactivity in tumor was lower than in blood and liver, excluding high-contrast imaging. In contrast, ${ }^{111}$ In-DOTA-Z09591 yielded a tumor-to-blood ratio of $28 \pm 14$, a tumor-to-liver ratio of $9 \pm 4$, and a tumor-to-bone ratio of $13 \pm 4$ at $2 \mathrm{~h}$ after injection, indicating that this tracer might be used for the detection of an increased level of PDGFR $\beta$ in all major metastatic sites by molecular imaging.

A unique cysteine at the $\mathrm{C}$ terminus of the Affibody molecule Z09591 may be applied for labeling using a variety of alternative radionuclides. In this study, thiol-directed chemistry was used for site-specific conjugation of the versatile DOTA chelator, which was used to chelate ${ }^{111} \mathrm{In}$. Radiolabeling did not affect the specificity of ${ }^{111}$ In-DOTA-Z09591 binding to PDGFR $\beta$-expressing
TABLE 4

Biodistribution of ${ }^{111}$ In-DOTA-Z09591 (Injected Dose $1 \mu \mathrm{g}$ ) at 1, 2, and 4 Hours After Injection in BALB/C nu/nu Mice Bearing U-87 MG Xenografts

\begin{tabular}{lccc}
\hline \multicolumn{1}{c}{ Organ } & $1 \mathrm{~h}$ & $2 \mathrm{~h}$ & $4 \mathrm{~h}$ \\
\hline Blood & $0.53 \pm 0.04^{*, \dagger}$ & $0.35 \pm 0.06^{\ddagger}$ & $0.20 \pm 0.07$ \\
Lung & $1.2 \pm 0.2^{\dagger}$ & $0.8 \pm 0.3^{\ddagger}$ & $0.3 \pm 0.1$ \\
Liver & $0.90 \pm 0.04^{*}$ & $0.80 \pm 0.07$ & $0.6 \pm 0.2$ \\
Spleen & $2.3 \pm 0.4^{\dagger}$ & $1.7 \pm 0.3^{\ddagger}$ & $1.0 \pm 0.2$ \\
Stomach & $1.3 \pm 0.1^{*, \dagger}$ & $1.0 \pm 0.2^{\ddagger}$ & $0.5 \pm 0.1$ \\
Colon & $1.8 \pm 0.4^{\dagger}$ & $1.3 \pm 0.2^{\ddagger}$ & $0.6 \pm 0.2$ \\
Kidney & $210 \pm 26^{*}$ & $249 \pm 15$ & $232 \pm 66$ \\
Tumor & $5.7 \pm 0.6^{\dagger}$ & $5.9 \pm 1.7$ & $4.0 \pm 0.8$ \\
Muscle & $0.61 \pm 0.09^{*, \dagger}$ & $0.34 \pm 0.03^{\ddagger}$ & $0.15 \pm 0.05$ \\
Bone & $0.79 \pm 0.03^{*, \dagger}$ & $0.56 \pm 0.06^{\ddagger}$ & $0.25 \pm 0.03$ \\
Gl tract $\$$ & $1.9 \pm 0.2^{*, \dagger}$ & $1.2 \pm 0.2^{\ddagger}$ & $0.8 \pm 0.2$
\end{tabular}

*Significant difference $(P<0.05)$ between 1 and $2 \mathrm{~h}$ after injection. ${ }^{\dagger}$ Significant difference $(P<0.05)$ between 1 and $4 \mathrm{~h}$ after injection. ${ }^{\ddagger}$ Significant difference $(P<0.05)$ between 2 and $4 \mathrm{~h}$ after injection.

$\S$ Data for GI tract are presented as \%ID per whole sample.

There was no significant difference between data for 2-h time point and data reported for injected dose of $1 \mu \mathrm{g}$ in Table 1. Data are presented as an average $\% \mathrm{ID} / \mathrm{g}$ and SD for 4 mice.

U-87 MG cells (Fig. 2A). The internalization of ${ }^{111}$ In-DOTA-Z09591 by U-87 MG was rather slow (Fig. 2C). This has been seen with Affibody molecules binding other receptors $(30,31)$. With PDGFR $\beta$, slow internalization may be due to the monovalent mode of binding of the Affibody molecule, because dimerization has been shown to drive endocytosis of PDGFR $\beta$ (35). With slow internalization, a strong binding to the cell surface target is required for obtaining high accumulation of radioactivity. The dissociation constant of ${ }^{111}$ In-DOTA-Z09591 for binding to U-87 MG cells was assessed as $92 \pm 10 \mathrm{pM}$. Previous studies suggest that a tracer displaying an affinity in this range meets the requirement for visualization of targets with relatively low expression and slow internalization rate (36). We used a residualizing ${ }^{111}$ In label in this first study, because it permits the detection of sites of uptake and catabolism in vivo.

TABLE 5

Tumor-to-Organ Ratios for ${ }^{111}$ In-DOTA-Z09591 Affibody

Molecule (Injected Dose $1 \mu \mathrm{g}$ ) at 1, 2, and 4 Hours After Injection in BALB/C nu/nu Mice Bearing U-87 MG Xenografts

\begin{tabular}{lccc}
\hline Organ & $1 \mathrm{~h}$ & $2 \mathrm{~h}$ & $4 \mathrm{~h}$ \\
\hline Blood & $11 \pm 1^{*, \dagger}$ & $17 \pm 3$ & $21 \pm 6$ \\
Lung & $5 \pm 1^{\dagger}$ & $8 \pm 3$ & $12 \pm 2$ \\
Liver & $6 \pm 1^{\dagger}$ & $8 \pm 3$ & $7 \pm 2$ \\
Spleen & $2.6 \pm 0.6^{\dagger}$ & $3.5 \pm 0.7$ & $4.2 \pm 0.4$ \\
Stomach & $4.4 \pm 0.5^{\star}, \dagger$ & $6.0 \pm 0.7$ & $8 \pm 1$ \\
Colon & $3.3 \pm 0.7^{\star, \dagger}$ & $4.6 \pm 0.7^{\ddagger}$ & $6.6 \pm 0.5$ \\
Muscle & $10 \pm 2^{\star}$, & $18 \pm 6$ & $30 \pm 14$ \\
Bone & $7 \pm 1^{\dagger}$ & $11 \pm 3$ & $16 \pm 3$
\end{tabular}

Data are presented as average of 4 animals and SD.

*Significant difference $(P<0.05)$ between 1 and $2 \mathrm{~h}$ after injection.

${ }^{\dagger}$ Significant difference $(P<0.05)$ between 1 and $4 \mathrm{~h}$ after injection.

${ }^{\ddagger}$ Significant difference $(P<0.05)$ between 2 and $4 \mathrm{~h}$ after injection. 


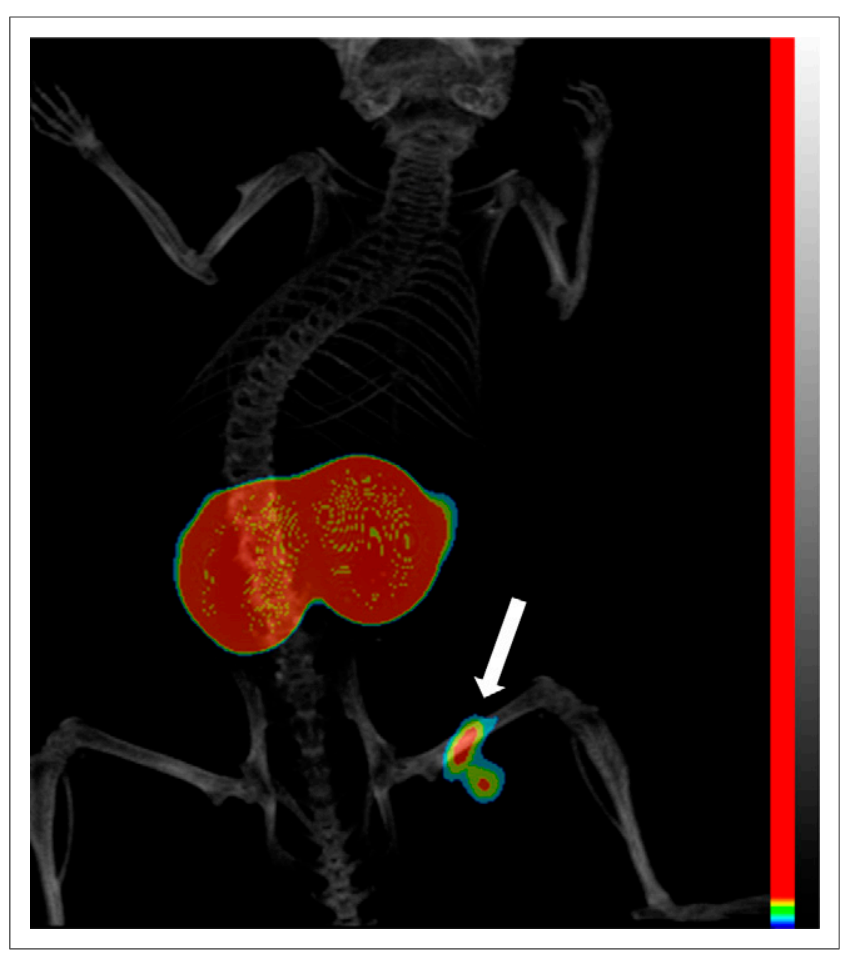

FIGURE 2. Imaging of PDFGR $\beta$ expression in U-87 MG xenograft (arrow) in BALB/C nu/nu mouse using small-animal SPECT/CT (maximumintensity projection). Image was acquired at $3 \mathrm{~h}$ after injection. SPECT scale, 0.02-0.04; CT scale, 400-2000 HU.

However, this was associated with a high renal retention of radioactivity. A slow internalization rate suggests that nonresidualizing labels, for example, radiohalogens or some forms of technetium labels, might be used in the future studies. Experience with derivatives of slowly internalizing HER2-binding Affibody molecules demonstrated that this enables a substantial reduction of renal retention of radioactivity without compromising tumor uptake $(37,38)$.

In humans, and probably also in mice, there is expression of PDGFR $\beta$ in normal organs and tissues (in normal vasculature and, according to Human Protein Atlas, http://www.proteinatlas.org/, in a subset of cells in the stomach, colon, and kidney). Therefore, we were concerned that it might be challenging to obtain a sufficient contrast for imaging the PDGFR $\beta$ overexpressing tumor in mice using a tracer that binds both human and murine PDGFR $\beta$. Increasing the injected dose of unlabeled protein may provide preferential block of binding to normal tissues with low antigen density (39). This requires that the receptor density is higher in the tumor, sufficiently high to avoid PDGFR $\beta$ saturation at a protein concentration providing low uptake in normal tissues. However, the expression level in U-87 MG cells is rather low (36,000 receptors/ cell). Earlier, we studied targeting of HER2-expressing LS174T xenografts, which display a target level similar to the level of PDGFR $\beta$ in the current tumor cell line U-87. With the monomeric HER2-binding Affibody molecule, an increase of the injected protein dose above $1 \mu \mathrm{g}$ per mouse was shown to cause an abrupt decrease of the tumor uptake (40). For this reason, the dose range tested in the present study was $0.1-1 \mu \mathrm{g}$ of protein. Except for the liver, no difference in accumulation of radioactivity in various tissues reached significance in this range (Table 1). However, tumorto-stomach, tumor-to-colon, and tumor-to-muscle ratios were higher after an injected dose of $1 \mu \mathrm{g}$ than after a dose of $0.1 \mu \mathrm{g}$. In contrast, increasing the protein dose to $70 \mu \mathrm{g}$ caused a significant decrease of uptake in all organs and tissues except for the kidney (Table 1; Supplemental Fig. 4). Tumor uptake was reduced more than 7-fold in comparison with the $1-\mu \mathrm{g}$ injected dose, which is taken as evidence of saturable binding of ${ }^{111}$ In-DOTA-Z09591 to PDGFR $\beta$ in the U-87 MG xenografts. The decrease of uptake in all organs and tissues except for the kidney indicated saturable binding to murine PDGFR $\beta$ receptors at these sites, likely including the vasculature. However, PDGFR $\beta$ is involved in the regulation of vascular permeability and is also expressed in glomeruli. The parental PDGFR $\beta$ binder Z02465 possesses some antagonistic properties, for example, it inhibits PDGF-B-induced phosphorylation of PDGFR $\beta$ (28). Therefore, interaction of large doses of DOTA-Z09591 with murine PDGFR $\beta$ might alter vascular permeability or glomerular filtration rate and influence the biodistribution of ${ }^{111}$ In-DOTAZ09591. If the organ uptake reduction is due to such effects, injection of $70 \mu \mathrm{g}$ of DOTA-Z09591 should have the same influence on the biodistribution of any Affibody molecule. However, the control experiment showed that $70 \mu \mathrm{g}$ of DOTA-Z09591 did not have any effect on the level of nonspecific uptake of the HER2binding ${ }^{111}$ In- DOTA-Z02395 in any tissue (Table 3). Taken together, these data support the hypothesis that the uptake of ${ }^{111} \mathrm{In}$ DOTA-Z09591 in various nontumor tissues was PDGFR $\beta$-specific.

There was a significant increase of the tumor-to-organ ratios between 1 and $2 \mathrm{~h}$ after injection. However, there was little difference in tumor-to-organ ratios between 2 and $4 \mathrm{~h}$ after injection (Table 5), suggesting that an optimal imaging time is between the later time points. This assumption is supported by clear visualization of the PDGFR $\beta$-expressing xenograft at $3 \mathrm{~h}$ after injection. This high contrast shortly after injection should enable the use of short-lived positron emitters, such as ${ }^{68} \mathrm{Ga}$ or ${ }^{18} \mathrm{~F}$, as labels. The use of PET might provide a further increase of imaging sensitivity and improve quantification accuracy.

\section{CONCLUSION}

This study has demonstrated the feasibility of in vivo targeting of PDGFR $\beta$-expressing xenografts using Affibody molecules. Further development of radiolabeled Affibody molecules might provide a useful clinical tool for imaging of PDGFR $\beta$ levels during various pathologic conditions.

\section{DISCLOSURE}

The costs of publication of this article were defrayed in part by the payment of page charges. Therefore, and solely to indicate this fact, this article is hereby marked "advertisement" in accordance with 18 USC section 1734 . This research was financially supported by grants from the Swedish Cancer Society (Cancerfonden) and Swedish Research Council (Vetenskapsrådet). Fredrik Y. Frejd and Per Jonasson are employees of Affibody AB. Affibody AB holds intellectual property for Affibody molecules. Academic partners have control of any data that might present a conflict of interest for employees or consultants. No other potential conflict of interest relevant to this article was reported.

\section{REFERENCES}

1. Fredriksson L, Li H, Eriksson U. The PDGF family: four gene products form five dimeric isoforms. Cytokine Growth Factor Rev. 2004;15:197-204.

2. Tallquist M, Kazlauskas A. PDGF signaling in cells and mice. Cytokine Growth Factor Rev. 2004;15:205-213.

3. Hellström M, Kalén M, Lindahl P, Abramsson A, Betsholtz C. Role of PDGF-B and PDGFR-beta in recruitment of vascular smooth muscle cells and pericytes 
during embryonic blood vessel formation in the mouse. Development. 1999; 126:3047-3055.

4. Östman A, Heldin CH. PDGF receptors as targets in tumor treatment. $A d v$ Cancer Res. 2007;97:247-274.

5. Pietras K, Sjoblom T, Rubin K, Heldin CH, Ostman A. PDGF receptors as cancer drug targets. Cancer Cell. 2003;3:439-443.

6. Paulsson J, Sjöblom T, Micke P, et al. Prognostic significance of stromal plateletderived growth factor beta-receptor expression in human breast cancer. Am J Pathol. 2009;175:334-341.

7. Hägglöf C, Hammarsten P, Josefsson A. Stromal PDGFRbeta expression in prostate tumors and non-malignant prostate tissue predicts prostate cancer survival. PLoS ONE. 2010;5:e10747.

8. Yonemori K, Tsuta K, Ando M, et al. Contrasting prognostic implications of platelet-derived growth factor receptor- $\beta$ and vascular endothelial growth factor receptor-2 in patients with angiosarcoma. Ann Surg Oncol. 2011;18:2841-2850.

9. Patel SH, Kneuertz PJ, Delgado M, et al. Clinically relevant biomarkers to select patients for targeted inhibitor therapy after resection of hepatocellular carcinoma. Ann Surg Oncol. 2011;18:3384-3390.

10. Donnem T, Andersen S, Al-Saad S, Al-Shibli K, Busund LT, Bremnes RM. Prognostic impact of angiogenic markers in non-small-cell lung cancer is related to tumor size. Clin Lung Cancer. 2011;12:106-115.

11. Laimer D, Dolznig H, Kollmann K, et al. PDGFR blockade is a rational and effective therapy for NPM-ALK-driven lymphomas. Nat Med. 2012;18:1699-1704.

12. Ruan J, Luo M, Wang C, et al. Imatinib disrupts lymphoma angiogenesis by targeting vascular pericytes. Blood. 2013;121:5192-5202.

13. Fingas CD, Mertens JC, Razumilava N, Bronk SF, Sirica AE, Gores GJ. Targeting PDGFR- $\beta$ in cholangiocarcinoma. Liver Int. 2012;32:400-409.

14. Yuasa T, Takahashi S, Hatake K, Yonese J, Fukui I. Biomarkers to predict response to sunitinib therapy and prognosis in metastatic renal cell cancer. Cancer Sci. 2011;102:1949-1957.

15. Van den Abbeele AD, Gatsonis C, de Vries DJ, et al. ACRIN 6665/RTOG 0132 phase II trial of neoadjuvant imatinib mesylate for operable malignant gastrointestinal stromal tumor: monitoring with ${ }^{18} \mathrm{~F}$-FDG PET and correlation with genotype and GLUT4 expression. J Nucl Med. 2012;53:567-574.

16. van Dongen GA, Poot AJ, Vugts DJ. PET imaging with radiolabeled antibodies and tyrosine kinase inhibitors: immuno-PET and TKI-PET. Tumour Biol. 2012; 33:607-615.

17. Slobbe P, Poot AJ, Windhorst AD, van Dongen GA. PET imaging with small-molecule tyrosine kinase inhibitors: TKI-PET. Drug Discov Today. 2012;17:1175-1187.

18. Tolmachev V, Stone-Elander S, Orlova A. Radiolabelled receptor-tyrosine-kinase targeting drugs for patient stratification and monitoring of therapy response: prospects and pitfalls. Lancet Oncol. 2010;11:992-1000.

19. Altai M, Tolmachev V, Orlova A. Radiolabelled probes targeting tyrosine-kinase receptors for personalized medicine. Curr Pharm Des. September 11, 2013 [Epub ahead of print].

20. Asakawa $\mathrm{C}$, Ogawa $\mathrm{M}$, Kumata $\mathrm{K}$, et al. $\left[{ }^{11} \mathrm{C}\right]$ Sorafenib: radiosynthesis and preliminary PET study of brain uptake in P-gp/BCRP knockout mice. Bioorg Med Chem Lett. 2011;21:2220-2223.

21. Wang JQ, Miller KD, Sledge GW, Zheng QH. Synthesis of $\left[{ }^{18} \mathrm{~F}\right] \mathrm{SU} 11248$, a new potential PET tracer for imaging cancer tyrosine kinase. Bioorg Med Chem Lett. 2005; 15:4380-4384.

22. Kil KE, Ding YS, Lin KS, et al. Synthesis and positron emission tomography studies of carbon-11-labeled Imatinib (Gleevec). Nucl Med Biol. 2007;34:153-163.
23. Gelovani JG. Molecular imaging of epidermal growth factor receptor expressionactivity at the kinase level in tumors with positron emission tomography. Cancer Metastasis Rev. 2008;27:645-653.

24. Ahlgren S, Tolmachev V. Radionuclide molecular imaging using Affibody molecules. Curr Pharm Biotechnol. 2010;11:581-589.

25. Tolmachev V. Imaging of HER-2 overexpression in tumors for guiding therapy. Curr Pharm Des. 2008;14:2999-3019.

26. Baum RP, Prasad V, Müller D, et al. Molecular imaging of HER2-expressing malignant tumors in breast cancer patients using synthetic ${ }^{111} \mathrm{In}$ - or ${ }^{68} \mathrm{Ga}$-labeled Affibody molecules. J Nucl Med. 2010;51:892-897.

27. Sandberg D, Wennborg A, Feldwisch F, et al. First clinical observations of HER2 specific [ $\left.{ }^{111} \mathrm{In}\right] \mathrm{ABY}-025$ metastatic detection capability in females with metastatic breast cancer [abstract]. J Nucl Med. 2012;53(suppl 1):220.

28. Lindborg M, Cortez E, Hoiden-Guthenberg I, et al. Engineered high-affinity Affibody molecules targeting platelet-derived growth factor receptor beta in vivo. J Mol Biol. 2011;407:298-315.

29. Feldwisch J, Tolmachev V. Engineering of Affibody molecules for therapy and diagnostics. Methods Mol Biol. 2012;899:103-126.

30. Ahlgren S, Orlova A, Rosik D, et al. Evaluation of maleimide derivative of DOTA for site-specific labeling of recombinant Affibody molecules. Bioconjug Chem. 2008;19:235-243.

31. Wållberg H, Orlova A. Slow internalization of anti-HER2 synthetic Affibody monomer ${ }^{111}$ In-DOTA-ZHER2:342-pep2: implications for development of labeled tracers. Cancer Biother Radiopharm. 2008;23:435-442.

32. Björke H, Andersson K. Automated, high-resolution cellular retention and uptake studies in vitro. Appl Radiat Isot. 2006;64:901-905.

33. Rajkumar VS, Boxer G, Robson M, Muddle J, Papastavrou Y, Pedley RB. A comparative study of PDGFR inhibition with imatinib on radiolabeled antibody targeting and clearance in two pathologically distinct models of colon adenocarcinoma. Tumour Biol. 2012;33:2019-2029.

34. Askoxylakis V, Marr A, Altmann A, et al. Peptide-based targeting of the plateletderived growth factor receptor beta. Mol Imaging Biol. 2013;15:212-221.

35. Pahara J, Shi H, Chen X, Wang Z. Dimerization drives PDGF receptor endocytosis through a C-terminal hydrophobic motif shared by EGF receptor. Exp Cell Res. 2010;316:2237-2250.

36. Tolmachev V, Tran TA, Rosik D, Abrahmsén L, Sjöberg A, Orlova A. Tumor targeting using Affibody molecules: interplay of affinity, target expression level and binding site composition. J Nucl Med. 2012;53:953-960.

37. Kramer-Marek G, Kiesewetter DO, Martiniova L, Jagoda E, Lee SB, Capala J. $\left[{ }^{18} \mathrm{~F}\right]$ FBEM-Z(HER2:342)-Affibody molecule-a new molecular tracer for in vivo monitoring of HER2 expression by positron emission tomography. Eur J Nucl Med Mol Imaging. 2008;35:1008-1018.

38. Wållberg $\mathrm{H}$, Orlova $\mathrm{A}$, Altai $\mathrm{M}$, et al. Molecular design and optimization of ${ }^{99 \mathrm{~m} T c-l a b e l e d ~ r e c o m b i n a n t}$ Affibody molecules improves their biodistribution and imaging properties. J Nucl Med. 2011;52:461-469.

39. Tolmachev V, Rosik D, Wållberg $\mathrm{H}$, et al. Imaging of EGFR expression in murine xenografts using site-specifically labelled anti-EGFR ${ }^{111}$ In-DOTA-Z EGFR:2377 Affibody molecule: aspect of the injected tracer amount. Eur J Nucl Med Mol Imaging. 2010;37:613-622.

40. Tolmachev V, Wållberg H, Sandström M, Hansson M, Wennborg A, Orlova A. Optimal specific radioactivity of anti-HER2 Affibody molecules enables discrimination between xenografts with high and low HER2 expression levels. Eur $J$ Nucl Med Mol Imaging. 2011;38:531-539. 